\title{
Bifurcations at infinity, invariant algebraic surfaces, homoclinic and heteroclinic orbits and centers of a new Lorenz-like chaotic system
}

\author{
Márcio R. A. Gouveia • Marcelo Messias • \\ Claudio Pessoa
}

Received: 11 March 2015 / Accepted: 18 November 2015 / Published online: 1 December 2015

(C) Springer Science+Business Media Dordrecht 2015

\begin{abstract}
We present a global dynamical analysis of the following quadratic differential system $\dot{x}=a(y-x), \dot{y}=d y-x z, \dot{z}=-b z+f x^{2}+g x y$, where $(x, y, z) \in \mathbb{R}^{3}$ are the state variables and $a, b, d, f, g$ are real parameters. This system has been proposed as a new type of chaotic system, having additional complex dynamical properties to the well-known chaotic systems defined in $\mathbb{R}^{3}$, alike Lorenz, Rössler, Chen and other. By using the Poincaré compactification for a polynomial vector field in $\mathbb{R}^{3}$, we study the dynamics of this system on the Poincaré ball, showing that it undergoes interesting types of bifurcations at infinity. We also investigate the existence of first integrals and study the dynamical behavior of the system on the invariant algebraic surfaces defined by these first integrals, showing the existence of families of homoclinic and heteroclinic orbits and centers contained on these invariant surfaces.
\end{abstract}

M. R. A. Gouveia · C. Pessoa

Departamento de Matemática, Instituto de Biociências, Letras e Ciências Exatas - IBILCE, Univ Estadual Paulista (UNESP), São José do Rio Preto, SP, Brazil

e-mail: maralves@ibilce.unesp.br

C. Pessoa

e-mail: pessoa@ibilce.unesp.br

M. Messias ( $\square)$

Departamento de Matemática e Computação, Faculdade de Ciências e Tecnologia - FCT, Univ Estadual Paulista (UNESP), Presidente Prudente, SP, Brazil e-mail: marcelo@fct.unesp.br
Keywords Quadratic system · Poincaré compactification · Dynamics at infinity - First integral · Invariant algebraic surfaces $\cdot$ Homoclinic orbits $\cdot$ Heteroclinic orbits $\cdot$ Centers on $\mathbb{R}^{3}$

Mathematics Subject Classification Primary: 37C29 $\cdot$ 37D45 - Secondary: 37B55

\section{Introduction}

In this paper, we perform a global dynamical analysis of the differential system given by

$\dot{x}=a(y-x), \quad \dot{y}=d y-x z, \quad \dot{z}=-b z+f x^{2}+g x y$,

where $(x, y, z) \in \mathbb{R}^{3}$ are the state variables and $a, b, d, f, g$ are real parameters. System (1) has been proposed in [4] to find out some new kind of dynamics not found in the well-known Lorenz system, given by [9]

$\dot{x}=\sigma(y-x), \quad \dot{y}=r x-y-x z, \quad \dot{z}=-b z+x y$,

nor in the Lorenz-like systems which appear in the literature (Chen, Rössler, Lü, Rikitake, Shimizu-Morioka and other).

Since the publication of the seminal paper [9] in 1963, several papers have appeared in the literature concerning the dynamical properties of the Lorenz and Lorenz-like systems, as the complex dynamics of their 
solutions and the creation of strange attractors. Furthermore, some quadratic systems similar to system (2) also appeared, arising from physical models or proposed from an abstract point of view, which are shown to be chaotic $[4,10-12,14-17,19,20]$. More recently, a series of papers have been published which present global dynamical analysis of Lorenz and Lorenz-like systems, giving a contribution to the understanding of these complex kinds of differential systems in $\mathbb{R}^{3}$; see, for instance [5-8, 12,13].

In [4], the authors proposed and studied system (1) as a new chaotic system differing from the known systems considered in the literature. They studied by both rigorous theoretical analysis and numerical simulations the dynamical properties of system (1) for $a>0$ and $f, g \geq 0$, with $f+g>0$. In their first main result, they gave a local description of the qualitative behavior of system (1) solutions corresponding to $b<0, b=0, b>0$ and varying the other parameters; in theses cases, they provide the number of equilibrium points (one or three) and analyzed the local stability of them, showing the occurrence of Hopf bifurcation, pitchfork bifurcation and degenerate pitchfork bifurcation. They also proved the nonexistence of closed orbits neither homoclinic orbits and the existence of two heteroclinic orbits when the parameters satisfy the conditions $a>d$ and $b g>2 a(f+g)$. Several figures in the mentioned paper present numerical simulations which corroborate the obtained results and allow for determining the existence of a chaotic attractor for $(a, b, d, f, g)=(10,3,6,1,0)$. All the results presented in [4] illustrate the complex dynamical behavior of system (1), which is not topologically conjugated to the Lorenz system (2).

In this paper, we study some global dynamical aspects of system (1) aiming to complement the results stated in [4] and to give a contribution to the understanding of its complex dynamics. More precisely we give a complete description of its phase portrait at infinity via the Poincaré compactification, showing that the system undergoes interesting bifurcations at infinity as the parameters $f$ and $g$ are varied, as for instance the collapse of a center into a singularity with an infinity of homoclinic orbits. In order to complete the analysis of system (1) for $f+g>0$ presented in [4], we prove that in the case $b=0$ and $f+g=0$, system (1) has the family of invariant algebraic surfaces given by $-f x^{2}-2 a z=r, r \in \mathbb{R}$, in which are contained families of symmetric homoclinic or heteroclinic orbits surrounding centers, for certain parameter values. Here we are calling a center in $\mathbb{R}^{3}$ a singularity belonging to a two-dimensional invariant surface which is surrounded by an annulus of periodic orbits also contained in this invariant surface. It is well known that homoclinic and heteroclinic orbits play a fundamental role on the study of differential systems in $\mathbb{R}^{3}$ and their existence are in general very difficult to be analytically proved. In this way, the technique used here to prove the existence of these types of orbits for system (1), via the existence of invariant algebraic surfaces and the Hamiltonian structure of system (1) restricted to these surfaces, is a good tool which may be used to study other differential systems in $\mathbb{R}^{3}$, especially polynomial differential systems coming from applied problems in Physics and Engineering.

Beyond to complement the results presented in [4], the analysis of system (1) presented here makes part of a program aiming to describe global properties of quadratic three-dimensional differential systems defined in $\mathbb{R}^{3}$, which is being developed by several authors; see, for instance $[5-8,12,13]$. It is important to observe that these types of systems appear in the literature as mathematical models for several natural problems, coming from Physics, Biology and Engineering mathematical modeling; see, for instance, $[3,5,6,9,13,21]$ and references therein. Also, one has recently found that the chaotic dynamics of differential systems is a very useful tool which has great potentials for applications in many branches of science and technology, such as information and computer science, power system protection, biomedical system analysis, encryption and communication, electronic circuits and so on (see [4] and its references). In this way, it is worth all efforts intended to understand and clarify the complex dynamics of systems like (1).

The paper is organized as follows. In Sect. 2 for the sake of completeness, we give a summary of Poincaré compactification technique for polynomial vector fields in $\mathbb{R}^{3}$, which will help the reader to understand the other sections. In Sect. 3, we present the dynamics and bifurcations of system (1) at infinity. In Sect. 4, we show that for certain parameter values system (1) has a family of invariant algebraic surfaces and study its dynamics on these surfaces, proving in particular the existence of homoclinic and heteroclinic orbits and centers. Some concluding remarks are given in Sect. 5. 


\section{Poincaré compactification in $\mathbb{R}^{3}$}

As any polynomial vector field, system (1) can be extended to an analytical system defined on a closed ball $B$ of radius one, whose interior is diffeomorphic to $\mathbb{R}^{3}$ and its invariant boundary, the two-dimensional sphere $\mathbb{S}^{2}=\left\{(x, y, z) \mid x^{2}+y^{2}+z^{2}=1\right\}$, plays the role of the infinity. This ball is known as the Poincaré ball, since the technique for doing this extension is the Poincaré compactification for a polynomial vector field in $\mathbb{R}^{n}$, which is described in detail, for example, in $[2,18]$. The boundary of $B$ is called the Poincaré sphere and represents the points of $\mathbb{R}^{3}$ at infinity. Poincaré introduced this compactification for polynomial vector fields in $\mathbb{R}^{2}$, and its extension to $\mathbb{R}^{n}$ for $n>2$ can be found in $[2,18]$. In this section, we present a summary of the Poincaré compactification for polynomial vector fields in $\mathbb{R}^{3}$, which will be used in the next sections in order to study system (1) at infinity.

Consider in $\mathbb{R}^{3}$ the polynomial differential system

$\dot{x}=P^{1}(x, y, z), \quad \dot{y}=P^{2}(x, y, z), \quad \dot{z}=P^{3}(x, y, z)$,

or equivalently its associated polynomial vector field $X=\left(P^{1}, P^{2}, P^{3}\right)$. The degree $n$ of $X$ is defined as $n=\max \left\{\operatorname{deg}\left(P^{i}\right): i=1,2,3\right\}$. Let $\mathbb{S}^{3}=\{y=$ $\left.\left(y_{1}, y_{2}, y_{3}, y_{4}\right) \in \mathbb{R}^{4}:\|y\|=1\right\}$ be the unit sphere in $\mathbb{R}^{4}, \mathbb{S}_{+}=\left\{y \in \mathbb{S}^{3}: y_{4}>0\right\}$ and $\mathbb{S}_{-}=\left\{y \in \mathbb{S}^{3}:\right.$ $\left.y_{4}<0\right\}$ be the northern and southern hemispheres of $\mathbb{S}^{3}$, respectively. The tangent space to $\mathbb{S}^{3}$ at the point $y$ is denoted by $T_{y} \mathbb{S}^{3}$. Then the tangent space

$T_{(0,0,0,1)} \mathbb{S}^{3}=\left\{\left(x_{1}, x_{2}, x_{3}, 1\right) \in \mathbb{R}^{4}:\left(x_{1}, x_{2}, x_{3}\right) \in \mathbb{R}^{3}\right\}$

is identified with $\mathbb{R}^{3}$.

Now consider the central projections

$$
\begin{aligned}
f_{+}: \mathbb{R}^{3}= & T_{(0,0,0,1)} \mathbb{S}^{3} \longrightarrow \mathbb{S}_{+} \text {and } f_{-}: \mathbb{R}^{3} \\
& =T_{(0,0,0,1)} \mathbb{S}^{3} \longrightarrow \mathbb{S}_{-},
\end{aligned}
$$

defined by $f_{ \pm}(x)= \pm\left(x_{1}, x_{2}, x_{3}, 1\right) / \Delta x$, where $\Delta x=$ $\left(1+\sum_{i=1}^{3} x_{i}^{2}\right)^{1 / 2}$. Through these central projections, $\mathbb{R}^{3}$ is identified with the northern and southern hemispheres. The equator of $\mathbb{S}^{3}$ is $\mathbb{S}^{2}=\left\{y \in \mathbb{S}^{3}: y_{4}=0\right\}$. Clearly, $\mathbb{S}^{2}$ can be identified with the infinity of $\mathbb{R}^{3}$.

The maps $f_{+}$and $f_{-}$define two copies of $X$ on $\mathbb{S}^{3}$ : one $D f_{+} \circ X$ in the northern hemisphere and the other $D f_{-} \circ X$ in the southern one. Denote by $\bar{X}$ the vector field on $\mathbb{S}^{3} \backslash \mathbb{S}^{2}=\mathbb{S}_{+} \cup \mathbb{S}_{-}$which restricted to $\mathbb{S}_{+}$ coincides with $D f_{+} \circ X$ and restricted to $\mathbb{S}_{-}$coincides with $D f_{-} \circ X$.

Now we can extend analytically the vector field $\bar{X}(y)$ to the whole sphere $\mathbb{S}^{3}$ by $p(X)(y)=y_{4}^{n-1} \bar{X}(y)$. The extended vector field $p(X)$ is called the Poincaré compactification of $X$ on $\mathbb{S}^{3}$.

As $\mathbb{S}^{3}$ is a differentiable manifold in order to compute the expression for $p(X)$, we can consider the eight local charts $\left(U_{i}, F_{i}\right),\left(V_{i}, G_{i}\right)$, where $U_{i}=\{y \in$ $\left.\mathbb{S}^{3}: y_{i}>0\right\}$ and $V_{i}=\left\{y \in \mathbb{S}^{3}: y_{i}<0\right\}$ for $i=1,2,3,4$ and the diffeomorphisms $F_{i}: U_{i} \rightarrow$ $\mathbb{R}^{3}$ and $G_{i}: V_{i} \rightarrow \mathbb{R}^{3}$ for $i=1,2,3,4$ are the inverses of the central projections from the origin to the tangent hyperplanes at the points $( \pm 1,0,0,0)$, $(0, \pm 1,0,0),(0,0, \pm 1,0)$ and $(0,0,0, \pm 1)$, respectively. Let us make the computations on $U_{1}$. Suppose that the origin $(0,0,0,0)$, the point $\left(y_{1}, y_{2}, y_{3}, y_{4}\right) \in$ $\mathbb{S}^{3}$ and the point $\left(1, z_{1}, z_{2}, z_{3}\right)$ in the tangent hyperplane to $\mathbb{S}^{3}$ at $(1,0,0,0)$ are collinear. Then we have $1 / y_{1}=z_{1} / y_{2}=z_{2} / y_{3}=z_{3} / y_{4}$, and consequently, $F_{1}(y)=\left(y_{2} / y_{1}, y_{3} / y_{1}, y_{4} / y_{1}\right)=\left(z_{1}, z_{2}, z_{3}\right)$ defines the coordinates on $U_{1}$. As

$$
D F_{1}(y)=\left(\begin{array}{cccc}
-y_{2} / y_{1}^{2} & 1 / y_{1} & 0 & 0 \\
-y_{3} / y_{1}^{2} & 0 & 1 / y_{1} & 0 \\
-y_{4} / y_{1}^{2} & 0 & 0 & 1 / y_{1}
\end{array}\right)
$$

and $y_{4}^{n-1}=\left(z_{3} / \Delta z\right)^{n-1}$, the analytical vector field $p(X)$ in the local chart $U_{1}$ becomes

$\frac{z_{3}^{n}}{(\Delta z)^{n-1}}\left(-z_{1} P^{1}+P^{2},-z_{2} P^{1}+P^{3},-z_{3} P^{1}\right)$,

where $P^{i}=P^{i}\left(1 / z_{3}, z_{1} / z_{3}, z_{2} / z_{3}\right)$ and $\Delta z=$ $\left(1+\sum_{i=1}^{3} z_{i}^{2}\right)^{1 / 2}$.

In a similar way, we can deduce the expressions of $p(X)$ in $U_{2}$ and $U_{3}$. These are

$\frac{z_{3}^{n}}{(\Delta z)^{n-1}}\left(-z_{1} P^{2}+P^{1},-z_{2} P^{2}+P^{3},-z_{3} P^{2}\right)$,

where $P^{i}=P^{i}\left(z_{1} / z_{3}, 1 / z_{3}, z_{2} / z_{3}\right)$ in $U_{2}$, and

$\frac{z_{3}^{n}}{(\Delta z)^{n-1}}\left(-z_{1} P^{3}+P^{1},-z_{2} P^{3}+P^{2},-z_{3} P^{3}\right)$,

where $P^{i}=P^{i}\left(z_{1} / z_{3}, z_{2} / z_{3}, 1 / z_{3}\right)$ in $U_{3}$. 
The expression for $p(X)$ in $U_{4}$ is $z_{3}^{n+1}\left(P^{1}, P^{2}, P^{3}\right)$. The expression for $p(X)$ in the local chart $V_{i}$ is the same as in $U_{i}$ multiplied by $(-1)^{n-1}$, where $n$ is the degree of $\left(P^{1}, P^{2}, P^{3}\right)$.

When we work with the expression of the compactified vector field $p(X)$ in the local charts, we usually omit the factor $1 /(\Delta z)^{n-1}$. We can do that through a rescaling of the time variable, since $\Delta z>0$.

In what follows we shall work with the orthogonal projection of $p(X)$ from the closed northern hemisphere to $y_{4}=0$, we continue denoting this projected vector field by $p(X)$. Note that the projection of the closed northern hemisphere is a closed ball $B$ of radius one, whose interior is diffeomorphic to $\mathbb{R}^{3}$ and whose boundary $\mathbb{S}^{2}$ corresponds to the infinity of $\mathbb{R}^{3}$. Of course $p(X)$ is defined in the whole closed ball $B$ in such a way that the flow on the boundary is invariant. This new vector field on $B$ is usually called the Poincaré compactification of $X$, and $B$ is called the Poincaré ball.

Remark 1 All the points on the invariant sphere $\mathbb{S}^{2}$ at infinity in the coordinates of any local chart $U_{i}$ and $V_{i}$ have $z_{3}=0$. The points in the interior of the Poincaré ball, which is diffeomorphic to $\mathbb{R}^{3}$, are given in the local charts $U_{1}, U_{2}$ and $U_{3}$ by $z_{3}>0$ and in the local charts $V_{1}, V_{2}$ and $V_{3}$ by $z_{3}<0$. See Fig. 1 for an illustration of the Poincaré sphere and the local charts $U_{i}$ and $V_{i}$ with their orientation. When we perform the compactification presented in this section, we obtain six polynomial vector fields defined on the local charts $U_{i}$ and $V_{i}, i=1 \ldots 3$. Then we study these vector fields using again the Poincare compactification, now in $\mathbb{R}^{2}$, from which we obtain six vector fields defined on the Poincaré disk, whose border corresponds to the points at infinity of each local chart $U_{i}$ and $V_{i}, i=1 \ldots 3$. See Figs. 2, 3, 4 and 5.

\section{Dynamics and bifurcations of system (1) at infinity}

In this section, we study the dynamics of system (1) at infinity by using the Poincaré compactification presented in Sect. 2.

Compactification in the local charts $U_{1}$ and $V_{1}$. From the results presented in Sect. 2, we obtain the expression

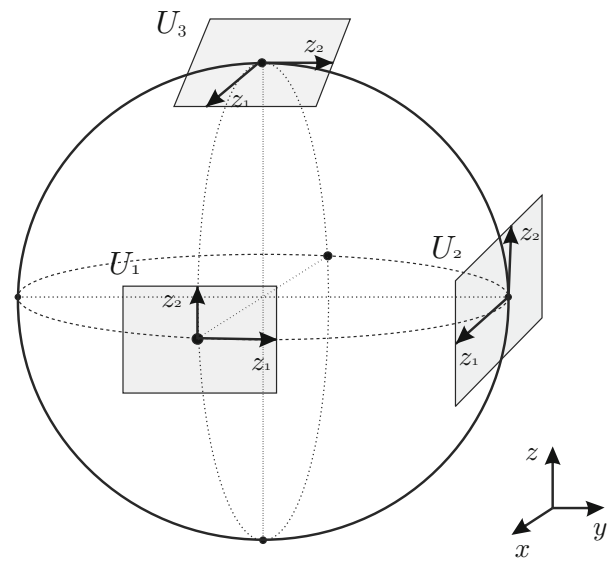

Fig. 1 Orientation of the local charts $U_{i}, i=1, \ldots, 3$ in the positive endpoints of the coordinate axis $x, y$ and $z$, used to draw the phase portrait of system (1) on the Poincaré sphere at infinity. The charts $V_{i} i=1, \ldots, 3$ are diametrically opposed to $U_{i}$, in the negative endpoints of the coordinate axis

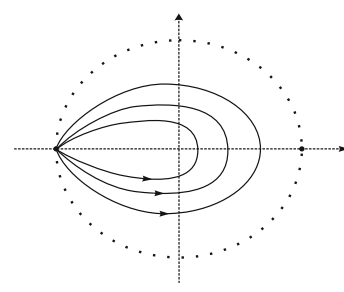

(a)

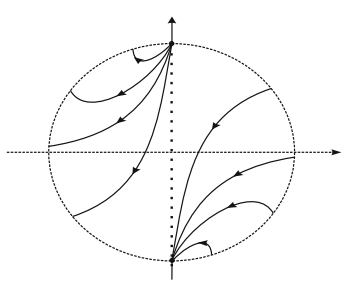

(c)

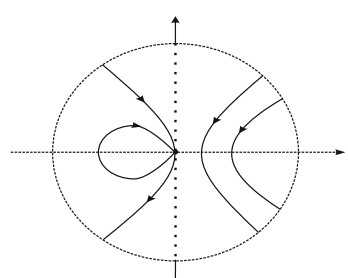

(b)

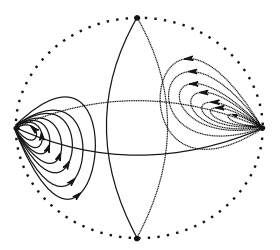

(d)
Fig. 2 Phase portrait of system (1) at infinity in the case $g=0$ and $f \neq 0$, in the local charts $U_{1}, U_{2}$ and $U_{3}$ (a, b c, respectively) and the global phase portrait on the Poincaré sphere (d)

of the Poincaré compactification $p(X)$ of system (1) in the local chart $U_{1}$, which is given by

$\dot{z}_{1}=-z_{2}+(a+d) z_{1} z_{3}-a z_{1}^{2} z_{3}$,

$\dot{z}_{2}=f+g z_{1}+(a-b) z_{2} z_{3}-a z_{1} z_{2} z_{3}$,

$\dot{z}_{3}=a z_{3}^{2}-a z_{1} z_{3}^{2}$.

Considering $z_{3}=0$ (which correspond to the points on the sphere $\mathbb{S}^{2}$ at infinity) system (3) reduces to 


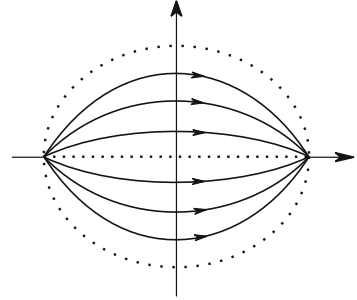

(a)

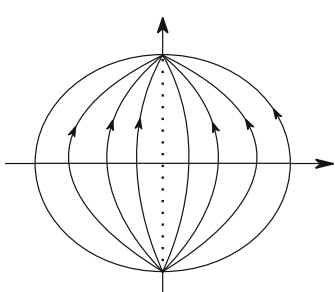

(c)

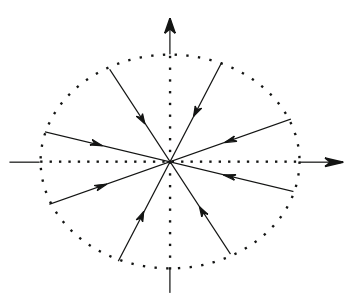

(b)

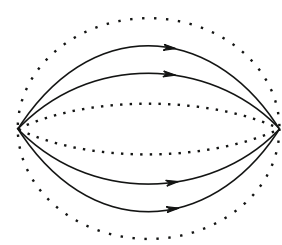

(d)
Fig. 3 Phase portrait of system (1) on the Poincaré sphere at infinity in the case $f=g=0$. a Chart $U_{1}$. b Chart $U_{2}$. c Chart $U_{3}$. d Poincaré sphere

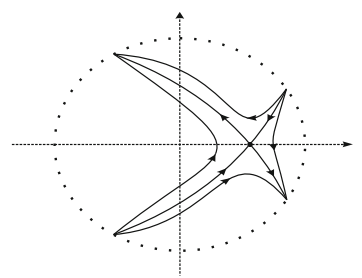

(a)

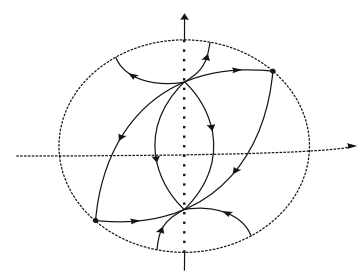

(c)

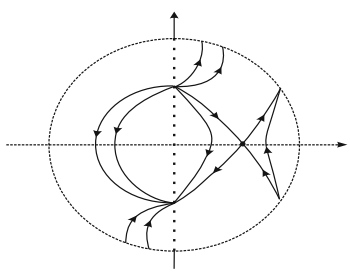

(b)

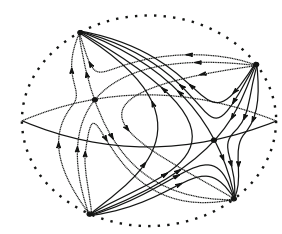

(d)
Fig. 4 Phase portrait of system (1) on the Poincaré sphere at infinity in the case $g<0$. a Chart $U_{1}$. b Chart $U_{2}$. c Chart $U_{3}$. d Poincaré sphere

$\dot{z}_{1}=-z_{2}, \quad \dot{z}_{2}=f+g z_{1}$,

which has no singularities if $g=0$ and $f \neq 0$. In this case, the phase portrait of system (4) on the Poincaré disk is as shown in Fig. 2a. Note that in this case there is a circle of singularities at infinity and an elliptic sector formed by infinite orbits homoclinic to the singular point $(-1,0)$ on the Poincaré disk. For $g \neq 0$, the system has the singularity $\left(z_{1}, z_{2}\right)=(-f / g, 0)$ which is

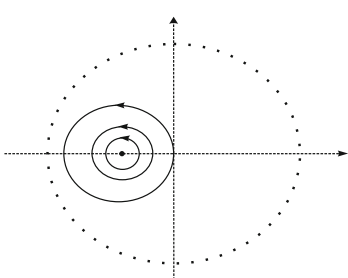

(a)

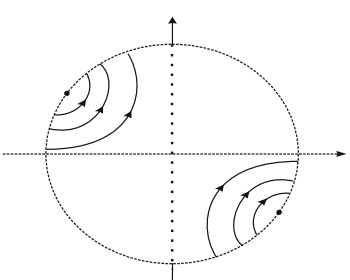

(c)

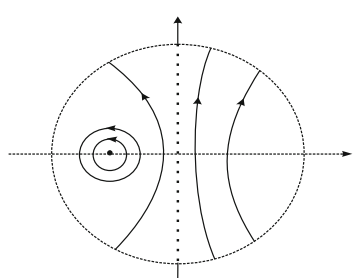

(b)

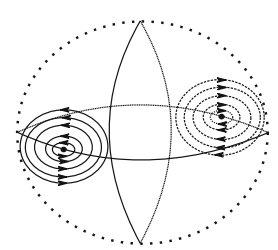

(d)
Fig. 5 Phase portrait of system (1) on the Poincare sphere at infinity in the case $g>0$. a Chart $U_{1}$. b Chart $U_{2}$. c Chart $U_{3}$. d Poincaré sphere

a saddle if $g<0$, see Fig. 4a, and a center if $g>0$, see Fig. 5a. For $g=f=0$, system (4) has a line of nonhyperbolic singularities; see Fig. 3a.

We observe that there is a difference between the phase portraits of system (1) at infinity given in this paper from the same type of phase portraits given for instance in $[5-8,12,13]$. Here the phase portrait at infinity in the local charts $U_{1}, U_{2}$ and $U_{3}$ is represented in the Poincaré disk. In this case, the points at infinity in one of the charts, given by the border $\mathbb{S}^{1}$ of the Poincaré disk, appear in the other chart. For example, the line of singularities at infinity of Fig. 2a with its elliptic sector appears in Fig. 2b as the $y$-axis. In this way, the global dynamics at infinity is easier to be represented and to be understood.

The phase portrait in the local chart $V_{1}$ is the same as the phase portrait in the local chart $U_{1}$ reversing the time, because the compactified vector field $p(X)$ in $V_{1}$ coincides with the vector field $p(X)$ in $U_{1}$ multiplied by -1 (for details, see Sect. 2).

Compactification in the local charts $U_{2}$ and $V_{2}$. Again using the results stated in Sect. 2, we obtain the expression of the Poincare compactification $p(X)$ of system (1) in the local chart $U_{2}$ as

$$
\begin{aligned}
& \dot{z}_{1}=a z_{3}-(d+a) z_{1} z_{3}+z_{1}^{2} z_{2}, \\
& \dot{z_{2}}=g z_{1}+f z_{1}^{2}-(d+b) z_{2} z_{3}+z_{1} z_{2}^{2}, \\
& \dot{z_{3}}=-d z_{3}^{2}-z_{1} z_{2} z_{3} .
\end{aligned}
$$


Taking $z_{3}=0$, system (5) reduces to

$\dot{z}_{1}=z_{1}^{2} z_{2}, \quad \dot{z}_{2}=g z_{1}+f z_{1}^{2}+z_{1} z_{2}^{2}$,

which has a line of singularities given by the $z_{2}$-axis. The linear part of system (6) at these singularities has null eigenvalues. Considering the invariance of $z_{1} z_{2}$ plane under the flow of (5), we can completely describe the dynamics on the sphere at infinity, in the cases $g=0$ and $f \neq 0 ; f=g=0 ; g<0$ and $f \in \mathbb{R} ; g>0$ and $f \in \mathbb{R}$, which are shown in Figs. 2b, 3b, $4 \mathrm{~b}$ and 5b, respectively.

The flow in the local chart $V_{2}$ is the same as the flow in the local chart $U_{2}$, because the compactified vector field $p(X)$ in $V_{2}$ coincides with the vector field $p(X)$ in $U_{2}$ multiplied by -1 . Hence, the phase portraits on the chart $V_{2}$ are the same shown in Figs. 2b, 3b, $4 \mathrm{~b}$ and $5 \mathrm{~b}$, reversing appropriately the time.

Compactification in the local charts $U_{3}$ and $V_{3}$. The expression of the Poincaré compactification $p(X)$ of system (1) in the local chart $U_{3}$ is

$\dot{z}_{1}=(b-a) z_{1} z_{3}+a z_{2} z_{3}-f z_{1}^{3}-g z_{1}^{2} z_{2}$,

$\dot{z}_{2}=-z_{1}+(b+d) z_{2} z_{3}-f z_{1}^{2} z_{2}-g z_{1} z_{2}^{2}$,

$\dot{z}_{3}=b z_{3}^{2}-f z_{1}^{2} z_{3}-g z_{1} z_{2} z_{3}$.

For $z_{3}=0$, system $(7)$ reduces to

$\dot{z}_{1}=-f z_{1}^{3}-g z_{1}^{2} z_{2}, \quad \dot{z}_{2}=-z_{1}-f z_{1}^{2} z_{2}-g z_{1} z_{2}^{2}$,

which also has a line of singularities given by the $z_{2}$-axis. Considering the invariance of $z_{1} z_{2}$-plane, we study the phase portraits of system (8), which corresponds to the phase portraits of system (1) at infinity on the chart $U_{3}$. They are shown in Figs. 2c, 3c, 4c and $5 c$.

Again the flow at infinity in the local chart $V_{3}$ is the same as the flow in the local chart $U_{3}$, reversing appropriately the time.

3.1 An interesting bifurcation at infinity: the collapse of a center into an infinity of petals

Considering the analysis made in the previous subsection and gluing the flows obtained in the six studied charts, shown in Figs. 2, 3, 4 and 5, we have a global picture of the dynamical behavior of system
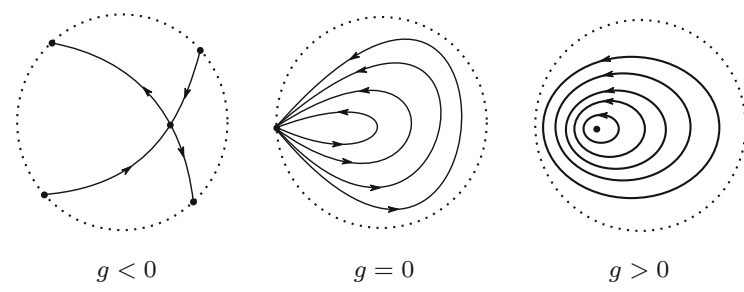

Fig. 6 Description of the bifurcation which occurs at infinity for $f \neq 0$ and the parameter $g$ varying in a neighborhood of zero: the collapse of a center into an infinity of petals

(1) on the Poincaré sphere at infinity. We can observe that some bifurcations occur at infinity, when the parameters $f$ and $g$ are varied. More precisely, assuming $f \neq 0$, the following bifurcation occurs. For $g>0$, the two symmetric centers in the equator of the Poincare sphere, shown in Fig. 5d, tend to the maximum cycle of singularities as the parameter $g$ goes to zero and becomes nonhyperbolic singularities with elliptic sectors, formed by infinitely many homoclinic orbits, or an infinity of petals, as shown in Fig. 2d; then for $g<0$, these nonhyperbolic singularities become hyperbolic saddles (see Fig. 4d). In Fig. 6, we illustrate this interesting type of bifurcation (see also Figs. 2, 4, 5).

It is important to note that in several studies presented in the literature about the dynamics at infinity of three-dimensional polynomial vector fields, as, for example, in the Lorenz system [12], no bifurcations at infinity occur. It is due to the fact that in the Lorenz system there is no parameter multiplying the quadratic terms in the equation, as it occurs with the parameters $f$ and $g$ of system (1) studied here. In fact, under the Poincaré compactification technique, only the largest degree terms of the polynomial vector field affect the dynamics at infinity. In this way, we can conclude that system (1) is actually nonequivalent to the Lorenz system (2), as stated in [4].

\section{Dynamics of system (1) on a family of invariant algebraic surfaces}

In this section, we provide a detailed description of system (1) dynamics with $b=0$ and $f+g=0$. In this case, this system reduces to

$$
\begin{aligned}
& \dot{x}=-a x+a y, \\
& \dot{y}=d y-x z, \\
& \dot{z}=f x^{2}-f x y .
\end{aligned}
$$


System (9) has two lines of singularities given by

$\{x=0, y=0, z=z\}, \quad\{x=y, y=y, z=d\}$.

Moreover, the function $F(x, y, z)=-f x^{2}-2 a z$ is a first integral of system (9), and consequently, it has $-f x^{2}-2 a z=r$, with $r \in \mathbb{R}$ as a family of invariant algebraic surfaces depending on the real parameter $r$. Hence, in order to study the phase portrait of system (9) we restricted its flow to these surfaces, since the phase space is folliated by them as the parameter $r$ is varied. The restricted system is given by

$\dot{x}=-a x+a y$,

$\dot{y}=\frac{r}{2 a} x+d y+\frac{f}{2 a} x^{3}$.

The divergent of system (11) is $d-a$, and hence, from Bendixson's Criterion it does not have limit cycles (see [1]). Moreover, if $d=a$ system (11) is a Hamiltonian system with Hamiltonian first integral

$H(x, y)=a x y-\frac{a}{2} y^{2}+\frac{r}{4 a} x^{2}+\frac{f}{8 a} x^{4}$.

System (11) has three singularities given by

$$
\left( \pm \frac{\sqrt{-f(r+2 a d)}}{f}, \pm \frac{\sqrt{-f(r+2 a d)}}{f}\right), \quad(0,0) .
$$

These singularities are obtained by the intersection of lines (10) with the invariant algebraic surface $-f x^{2}-$ $2 a z=r$, so the first ones are defined if $f<0$ and $r>-2 a d$, or if $f>0$ and $r<-2 a d$.

As system (1) is invariant under the change of variables $(x, y, z) \mapsto(-x,-y, z)$, i.e., it is symmetric with respect to the $z$-axis, in order to study the local stability we can consider only the singularities

$$
\left(\frac{\sqrt{-f(r+2 a d)}}{f}, \frac{\sqrt{-f(r+2 a d)}}{f}\right),(0,0) .
$$

The eigenvalues of the linear part of system (11) at the origin are given by

$$
\frac{d-a}{2} \pm \frac{\sqrt{(d-a)^{2}-4\left(-d a-\frac{r}{2}\right)}}{2} .
$$

Then the origin is: a saddle if $r>-2 a d$; a node if $-\frac{(a+d)^{2}}{2}<r<-2 a d$; a focus if $d \neq a$ and $r<$ $-\frac{(a+d)^{2}}{2}$; or a center if $d=a$ and $r<-\frac{(a+d)^{2}}{2}=$ $-2 a^{2}$.
Now if the trace of the linear part of system (11) at the origin is not null and its determinant is null, i.e., $d \neq a$ and $r=-2 a d$, system (11) becomes

$\dot{x}=-a x+a y$,

$\dot{y}=-d x+d y+\frac{f}{2 a} x^{3}$;

Doing the change of variables $(x, y) \mapsto\left(\frac{a}{d} u+v, u+v\right)$ and introducing the new independent variable given by $d t=(d-a) d s$, system (15) becomes

$\dot{u}=u+P(u, v)$,

$\dot{v}=Q(u, v)$,

where

$$
\begin{aligned}
P(u, v)= & -\frac{a^{2} f}{2 d^{2}(d-a)^{2}} u^{3}-\frac{3 f a}{2 d(a-d)^{2}} v u^{2} \\
& -\frac{3 f}{2(a-d)^{2}} u v^{2}-\frac{d f}{2 a(d-a)^{2}} v^{3}
\end{aligned}
$$

and

$$
\begin{aligned}
Q(u, v)= & \frac{a^{3} f}{2 d^{3}(d-a)^{2}} u^{3}+\frac{3 a^{2} f}{2 d^{2}(d-a)^{2}} v u^{2} \\
& +\frac{3 f a}{2 d(d-a)^{2}} u v^{2}+\frac{f}{2(d-a)^{2}} v^{3} .
\end{aligned}
$$

If $\varphi(v)$ is the solution of the equation $u+P(u, v)=0$, then

$\varphi(v)=-\frac{f d}{2 a(d-a)^{2}} v^{3}+\mathcal{O}\left(v^{4}\right)$,

and

$Q(\varphi(v), v)=\frac{f}{2(a-d)} v^{3}+\mathcal{O}\left(v^{5}\right)$.

Hence by Theorem 65, page 340 of [1], the origin is either a node if $\frac{f}{2(d-a)}>0$ or a saddle if $\frac{f}{2(d-a)}<0$. Moreover, if $f=0$ then the straight line $y=x$ is a line of singularities of system (11). In the case $d=0$, the same Theorem 65 [1] ensures that the origin is either a node if $-\frac{f}{2 a^{2}}>0$ or a saddle if $-\frac{f}{2 a^{2}}<0$.

For the case $a=d$ and $r=-2 a d$, i.e., the linear part of system (11) has trace and determinant equal to zero, system (15) becomes, after a rescaling of the time variable,

$\dot{x}=-x+y$,

$\dot{y}=-x+y+\frac{f}{2 a^{2}} x^{3}$.

Introducing the new variables $(u, v)$ given by $(x, y) \mapsto$ $(u+v, u+2 v)$, system (17) becomes

$\dot{u}=v+\tilde{P}(u, v)$,

$\dot{v}=\tilde{Q}(u, v)$, 
where

$\tilde{P}(u, v)=-\frac{f}{2 a^{2}} u^{3}-\frac{3 f}{2 a^{2}} v u^{2}-\frac{3 f}{2 a^{2}} u v^{2}-\frac{f}{2 a^{2}} v^{3}$

and

$\tilde{Q}(u, v)=\frac{f}{2 a^{2}} u^{3}+\frac{3 f}{2 a^{2}} v u^{2}+\frac{3 f}{2 a^{2}} u v^{2}+\frac{f}{2 a^{2}} v^{3}$.

If $\varphi(u)$ is the solution of the equation $v+\tilde{P}(u, v)=0$, then

$$
\begin{aligned}
\varphi(u) & =\frac{f}{2 a^{2}} u^{3}+\mathcal{O}\left(u^{5}\right), \text { and } \tilde{Q}(u, \varphi(u)) \\
& =\frac{f}{2 a^{2}} u^{3}+\mathcal{O}\left(v^{5}\right) .
\end{aligned}
$$

Hence, as the divergent of system (18) is null, by Theorem 66, page 357 of [1], the origin is either a saddle if $f>0$ or a center if $f<0$. Moreover, as $a \neq 0$, if $f=0$ then the straight line $y=x$ is a line of singularities of system (11).

The eigenvalues of the linear part of system (11) at the singularity $\left(\frac{\sqrt{-f(r+2 a d)}}{f}, \frac{\sqrt{-f(r+2 a d)}}{f}\right)$ are given by

$\frac{d-a}{2} \pm \frac{\sqrt{(d-a)^{2}-4(r+2 a d)}}{2}$.

For $f>0$ and $r<-2 a d$, this singularity is a saddle. For $f<0, r>-2 a d$ and $a \neq d$ then the singularity is either a node if $r \leq \frac{(d-a)^{2}}{4}-2 a d$ or a focus if $r>\frac{(d-a)^{2}}{4}-2 a d$. When $a=d$ the singularity is a center, because the divergent of system (11) is zero, i.e., the system is integrable.

We can summarize the above discussion in the following theorems, which give a complete characterization of the phase portraits of system (11), which is the restriction of system (1) with $b=0$ and $f=-g$ on the invariant algebraic surfaces $-f x^{2}-2 a z=r, \quad r \in \mathbb{R}$.

Theorem 1 Consider system (11) with $f=0$. Then the invariant algebraic surfaces reduce to the planes $2 a z=-r, r \in \mathbb{R}$ and the following results hold.

(i) If $d \neq a$, then:

(a) the origin is the unique singularity of system (11) which is a saddle if $r>-2 a d$ (see Fig. 7a);

(b) the origin is the unique singularity of system (11) which is a node if $-\frac{(a+d)^{2}}{2} \leq r<-2 a d$ (see Fig. 7b);

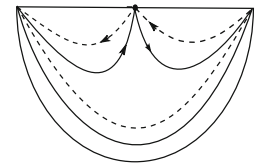

(a)

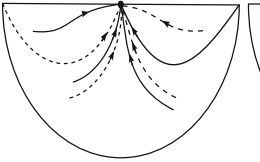

(b)

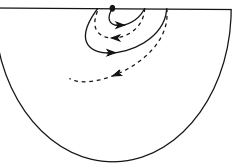

(c)
Fig. 7 Phase portraits of system (11) with $f=0$ on the invariant algebraic plane $-2 a z=r: \mathbf{a} r>-2 a d ; \mathbf{b}-\frac{(a+d)^{2}}{2} \leq r<$ $-2 a d ;$ and $\mathbf{c} r<-\frac{(a+d)^{2}}{2}$

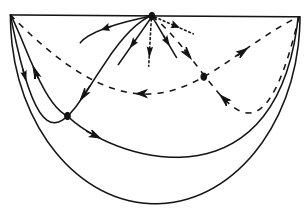

(a)

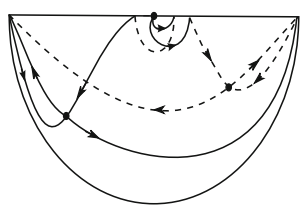

(b)
Fig. 8 Phase portraits of system (11) with $f>0$ on the invariant algebraic surface $-f x^{2}-2 a z=r: \mathbf{a} r>-\frac{(a+d)^{2}}{2}$ or $r=$ $-\frac{(a+d)^{2}}{2}$ and $d \neq a ; \quad \mathbf{b} r<-\frac{(a+d)^{2}}{2}$ and $d \neq a$

(c) the origin is the unique singularity of system (11) which is a focus if $r<-\frac{(a+d)^{2}}{2}$ (see Fig. 7c);

(ii) If $r=-2 a d$ then the straight line $y=x$ is a line of singularities of system (11).

Theorem 2 Consider system (11) with $f>0$. The following results hold.

(i) If $r>-2 a d$, then the origin is the unique singularity which is a saddle (see Fig. 7a);

(ii) If $r<-2 a d$, then the singularities $\left( \pm \frac{\sqrt{-f(r+2 a d)}}{f}\right.$, $\left.\pm \frac{\sqrt{-f(r+2 a d)}}{f}\right)$ are saddles and

(a) the origin is a node if either $r>-\frac{(a+d)^{2}}{2}$ or $r=-\frac{(a+d)^{2}}{2}$ and $d \neq a$ (see Fig. $8 \mathrm{a}$ );

(b) the origin is a focus if $r<-\frac{(a+d)^{2}}{2}$ and $d \neq a$ (see Fig. 8b);

(iii) If $a-d>0, d \neq 0$ and $r=-2 a d$, then the origin is the unique singularity which is a node (see Fig. 7b);

(iv) If $a-d<0, d \neq 0$ and $r=-2 a d$, then the origin is the unique singularity which is a saddle (see Fig. 7a);

(v) If $d=0$ and $r=0$, then the origin is the unique singularity which is a saddle (see Fig. 7a); 


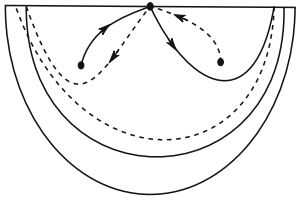

(a)

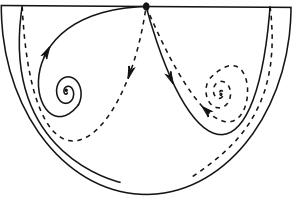

(b)
Fig. 9 Phase portraits of system (11) with $f<0$ on the invariant algebraic surface $-f x^{2}-2 a z=r: \mathbf{a}-2 a d<r \leq \frac{(d-a)^{2}}{4}-2 a d$; b $r>\frac{(d-a)^{2}}{4}-2 a d$

Theorem 3 Consider system (11) with $f<0$. The following results hold.

(i) If $-2 a d<r \leq \frac{(d-a)^{2}}{4}-2 a d$, then the origin is a saddle and the singularities $\left( \pm \frac{\sqrt{-f(r+2 a d)}}{f}\right.$, $\left.\pm \frac{\sqrt{-f(r+2 a d)}}{f}\right)$ are nodes (see Fig. 9a);

(ii) If $r>\frac{(d-a)^{2}}{4}-2 a d$, then the origin is a saddle and the singularities

$$
\left( \pm \frac{\sqrt{-f(r+2 a d)}}{f}, \pm \frac{\sqrt{-f(r+2 a d)}}{f}\right)
$$

are foci (see Fig. 9b);

(iii) If $r<-2 a d$, then the origin is the unique singularity, which is

(a) a node if either $r>-\frac{(a+d)^{2}}{2}$ or $r=-\frac{(a+d)^{2}}{2}$ and $d \neq a$ (see Fig. 7b);

(b) a focus if $\neq a$ and $r<-\frac{(a+d)^{2}}{2}$ (see Fig. 7c);

(v) If $d \neq 0$ and $r=-2 a d$, then the origin is the unique singularity which is a node if $a-d<0$ (see Fig. 7b) and a saddle if $a-d>0$ (see Fig. 7a);

(vi) If $d=0$ and $r=0$, then the origin is the unique singularity which is a node (see Fig. 7b);

Now, due to the fact that for $a=d$ system (11) is Hamiltonian, using the properties of the Hamiltonian first integral (12) and the discussion made above about the stability of singularities of this system, we can state the following theorem.

Theorem 4 Consider system (11) with $a=d$. In this case, the system has the Hamiltonian first integral (12) and the following results hold.

(i) If $f=0$, then the invariant algebraic surface reduces to the plane $2 a z=-r$ and the origin is the unique singularity of system (11) which is a center

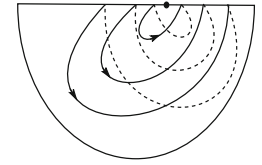

(a)

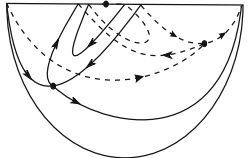

(b)

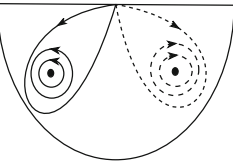

(c)
Fig. 10 Phase portraits of system (11) with $f=0$ on the invariant algebraic surface $-f x^{2}-2 a z=r$ : a the origin is a center; $\mathbf{b}$ the existence of heteroclinic orbits; $\mathbf{c}$ the existence of homoclinic orbits

if $r<-2 a^{2}$ (see Fig. 10a) or a saddle if $r>-2 a^{2}$ (see Fig. 7a);

(ii) If $f>0$ and $r<-2 a^{2}$, then the origin is a center and there exist two heteroclinic orbits connecting the singularities $\left( \pm \frac{\sqrt{-f\left(r+2 a^{2}\right)}}{f}, \pm \frac{\sqrt{-f\left(r+2 a^{2}\right)}}{f}\right)$, which are saddles (see Fig. 10b);

(iii) If $f>0$ and $r \geq-2 a^{2}$, then the origin is the unique singularity which is a saddle (see Fig. 7a);

(iv) If $f<0$ and $r>-2 a^{2}$, then the origin is a saddle, the singularities

$\left( \pm \frac{\sqrt{-f\left(r+2 a^{2}\right)}}{f}, \pm \frac{\sqrt{-f\left(r+2 a^{2}\right)}}{f}\right)$

are centers and there are two symmetric homoclinic orbits to the origin, surrounding these centers (see Fig. 10c);

(v) If $f<0$ and $r \leq-2 a^{2}$, then the origin is the unique singularity, which is a center (see Fig. 10a).

Remark 2 (i) The homoclinic and heteroclinic connections of system (11) described in statements (ii) and (iv) of Theorem 4 and shown in Fig. 10b, c are defined by the zero-level curves of the Hamiltonian function

$H(x, y)=a x y-\frac{a}{2} y^{2}+\frac{r}{4 a} x^{2}+\frac{f}{8 a} x^{4}$,

in each corresponding case. The saddles are determined by the maximum points, while the centers are determined by the minimum points of $H$.

(ii) From the results stated in Theorem 4, we can draw the bifurcation diagram of system (11), depending on the parameters $a$ and $r$, according to the signal of $f$ and the parabola $r=-2 a^{2}$, which is shown in Fig. 11.

(iii) In Fig. 12, we present some numerical simulations showing the family of symmetric homoclinic orbits contained on the invariant algebraic surfaces $-f x^{2}-2 a z=r$. 
Fig. 11 Bifurcation diagram of system (11) depending on the parameters $a$ and $r: \mathbf{a}$ $f<0$; b $f=0$; c $f>0$

Fig. 12 a Symmetric homoclinic orbits of system (9) contained on the invariant algebraic surfaces $-f x^{2}-2 a z=r$ and their projections: $\mathbf{b}$ on the plane $x z ; \mathbf{c}$ on the plane $x y$

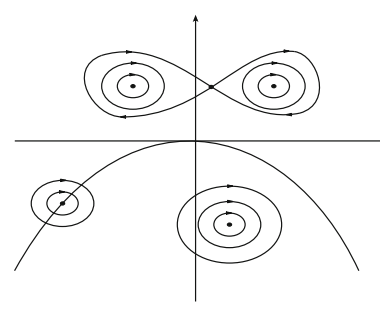

(a)

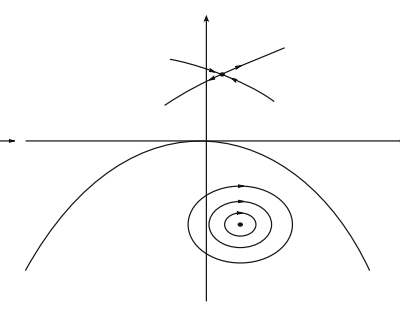

(b)

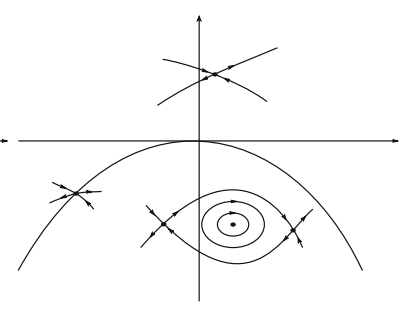

(c)

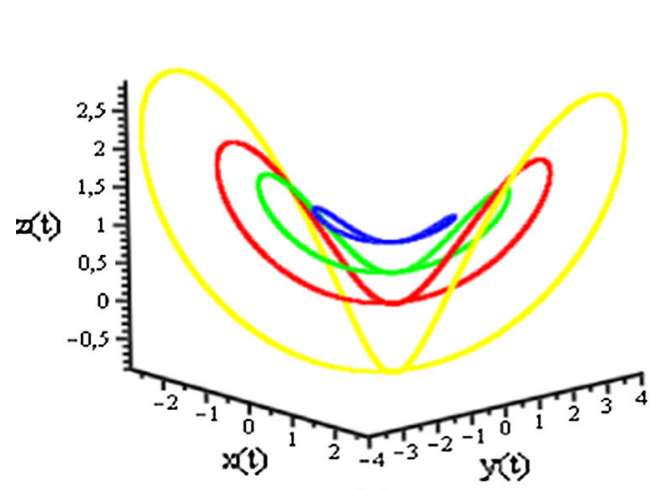

(a)

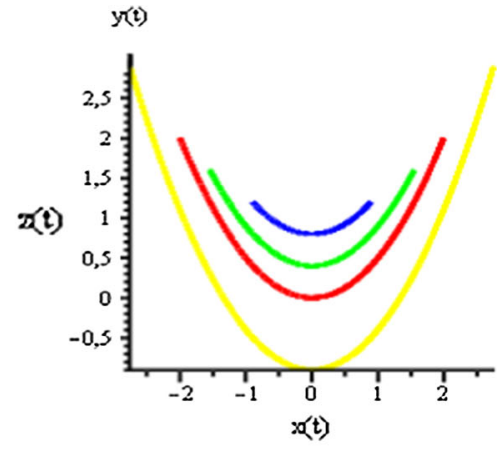

(b)

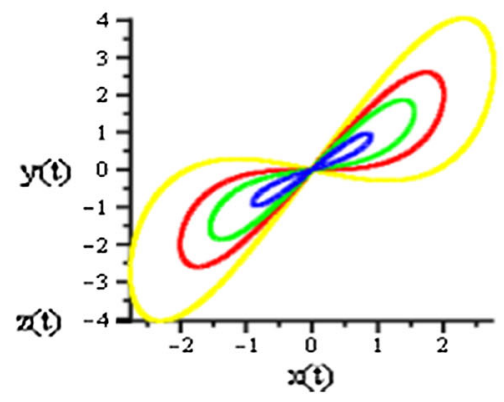

(c)

\section{Concluding remarks}

In this paper, we studied the dynamics and bifurcations of system (1) at infinity using the Poincaré compactification for a polynomial vector field in $\mathbb{R}^{3}$. We show that it undergoes interesting types of bifurcations at infinity as the parameters $f$ and $g$ are varied, different from the Lorenz and other chaotic systems, which has no these types of bifurcations $[5-8,12,13]$. This confirms that system (1) is actually a new chaotic quadratic Lorenzlike system, which is not equivalent to the Lorenz system. We also prove that in the case $b=0$ and $f+g=0$, system (1) has the family of invariant algebraic surfaces given by $-f x^{2}-2 a z=r, r \in \mathbb{R}$. We give a complete description of system (1) dynamics restricted to this family of invariant surfaces for different ranges of the parameter values. While in [4] the authors show that for $a>d$ and $b g>2 a(f+g)$ system (1) has no periodic nor homoclinic orbits and has only two heteroclinic orbits, here using the Hamiltonian structure of system (1) restricted to the invariant surfaces $-f x^{2}-2 a z=$ $r, r \in \mathbb{R}$, we prove that for $b=0, a=d$ and $f+g=0$ there exist families of symmetric homoclinic and heteroclinic orbits surrounding centers, contained in such invariant surfaces. We observe that, in this case, system (1) does not present chaotic dynamics, since the phase space is folliated by the invariant parabolic cylinders $-f x^{2}-2 a z=r, \quad r \in \mathbb{R}$, and hence, the dynamics is essentially two-dimensional. The results obtained in this work complement the ones presented in [4], where 
the same system (1) was studied, since beyond the dynamics and bifurcations at infinity studied here we also consider different ranges of variation for the parameters, not considered in [4] and for which different and interesting types of dynamics occur.

Acknowledgments The first and third authors were partially supported by CAPES and FAPESP. The second author was supported by FAPESP Projects 2012/18413-7 and 2013/24541-0 and by CNPq Project 308315/2012-0. The authors would like to thank the referees for their valuable comments and suggestions, which enabled them to improve the presentation of the paper.

\section{References}

1. Andronov, A.A., Leontovich, E.A., Gordon, I.I., Maier, A.G.: Qualitative Theory of Second Order Dynamic Systems. Wiley, Israel (1973)

2. Cima, A., Llibre, J.: Bounded polynomial vector fields. Trans. Am. Math. Soc. 318, 557-579 (1990)

3. Guckenheimer, J., Holmes, P.: Nonlinear Oscillations, Dynamical Systems, and Bifurcations of Vector Fields 7th printing. Springer, New York (2002)

4. Li, X., Ou, Q.: Dynamical properties and simulation of a new Lorenz-like chaotic system. Nonlinear Dyn. 65, 255$270(2011)$

5. Llibre, J., Messias, M.: Global dynamics of the Rikitake system. Phys. D 238(3), 241-252 (2009)

6. Llibre, J., Messias, M., da Silva, P.R.: On the global dynamics of the Rabinovich system. J. Phys. A Math. Theor. 41, 275210 (2008). (21pp)

7. Llibre, J., Messias, M., da Silva, P.R.: Global dynamics of the Lorenz system with invariant algebraic surfaces. Int. J. Bifurcat. Chaos Appl. Sci. Eng. 20(10), 3137-3155 (2010)

8. Llibre, J., Messias, M., da Silva, P.R.: Global dynamics in the Poincaré ball of the Chen system having invariant algebraic surfaces. Int. J. Bifurcat. Chaos Appl. Sci. Eng. 22(6), 1250154 (2012). (17 pages)
9. Lorenz, E.N.: Deterministic nonperiodic flow. J. Atmos. Sci. 20, 130-141 (1963)

10. Lü, J., Chen, G., Cheng, D.: A new chaotic system and beyond: the generalized Lorenz-like system. Int. J. Bifurc. Chaos Appl. Sci. Eng. 14(5), 1507-1537 (2004)

11. Mello, L.F., Messias, M., Braga, D.C.: Bifurcation analysis of a new Lorenz-like chaotic system. Chaos Solitons Fractals 37, 1244-1255 (2008)

12. Messias, M.: Dynamics at infinity and the existence of singularly degenerate heteroclinic cycles in the Lorenz system. J. Phys. A Math. Theor. 42, 115101 (2009)

13. Messias, M., Gouveia, M.A., Pessoa, C.: Dynamics at infinity and other global dynamical aspects of Shimizu-Morioka equations. Nonlinear Dyn. 69, 577-587 (2012)

14. Messias, M., Nespoli, C., Dalbelo, T.M.: Mechanics for the creation of strange attractors in Rössler's second system (Portuguese). TEMA Tend. Mat. Apl. Comput 9(2), 275285 (2008)

15. Shilnikov, A.L.: On bifurcations of the Lorenz attractor in the Shimizu-Morioka model. Phys. D 62, 338-346 (1993)

16. Shimizu, T., Morioka, N.: On the bifurcation of a symmetric limit cycle to an asymmetric one in a simple model. Phys. Lett. A 76(3,4), 201-204 (1980)

17. Tigan, G., Turaev, D.: Analytical search for homoclinic bifurcations in the Shimizu-Morioka model. Phys. D 240(12), 985-989 (2011)

18. Velasco, E.A.G.: Generic properties of polynomial vector fields at infinity. Trans. Am. Math. Soc. 143, 201-221 (1969)

19. Vladimirov, A.G., Volkov, D.Y.: Low-intensity chaotic operations of a laser with a saturable absorber. Opt. Commun. 100, 351-360 (1993)

20. Yu, S., Tang, W.K.S., Lü, J., Chen, G.: Generation of $n \times$ $m$-wing Lorenz-like attractors from a modified ShimizuMorioka model. IEEE Trans. Circuits Syst. 55(11), 1168$1172(2008)$

21. Wiggins, S.: Introduction to Applied Nonlinear Dynamical Systems and Chaos, 2nd edn. Springer, New York (2003) 